Check for updates

Cite this: Nanoscale Adv., 2019, 1, 1186

\title{
Influence of interlayer interactions on the relaxation dynamics of excitons in ultrathin $\mathrm{MoS}_{2}$
}

\author{
Dongxiao Lu, (D) ab Qiang Zhou, ${ }^{\text {*a }}$ Fangfei Li, ${ }^{a}$ Xiaowei Li (D) ${ }^{c}$ and Geyu Lu*b
}

Interlayer interactions play a crucial role in modifying the optical and electronic properties of layered materials in a complex way, which is of key importance for the performance of the optoelectronic devices based on these novel materials. In this contribution, we performed an investigation into the underlying influence of interlayer interactions on the relaxation dynamics of excitons in ultrathin $\mathrm{MoS}_{2}$ using the femtosecond transient absorption spectroscopy technique. The experimental results manifest that interlayer interactions in bilayer $\mathrm{MoS}_{2}$ can largely facilitate the exciton-phonon scattering process and inhibit the radiative recombination process, which consequently accelerates the relaxation rate of $A$ excitons and results in the decrease of the relaxation lifetime of A excitons in bilayer $\mathrm{MoS}_{2}$

Received 12th July 2018

Accepted 17th December 2018

DOI: $10.1039 / \mathrm{c} 8 \mathrm{na00086g}$

rsc.li/nanoscale-advances

\section{Introduction}

Two-dimensional layered transition-metal dichalcogenides (TMDs) have attracted growing attention in recent decades due to their remarkable optical and electronic properties. Unlike the zero band gap of graphene, TMDs have potential advantages in optoelectronic devices in virtue of their intrinsic semiconductor characteristics with a sizable band gap. As a typical TMD semiconductor with a bandgap of 1.2-2 $\mathrm{eV}$, molybdenum disulphide $\left(\mathrm{MoS}_{2}\right)$ exhibits fascinating optical properties, such as strong exciton luminescence, ${ }^{\mathbf{1 , 2}}$ large exciton binding energy, ${ }^{3,4}$ and valley polarization. ${ }^{5,6}$ As such, $\mathrm{MoS}_{2}$ has been widely applied in light emitting diodes, ${ }^{7-9}$ photodiodes, ${ }^{\mathbf{1 0}-12}$ solar cells, ${ }^{\mathbf{1 3 - 1 5}}$ and field effect transistors. ${ }^{16-18}$ It is generally known that each molecular layer of $\mathrm{MoS}_{2}$ is constructed with a molybdenum-atom layer sandwiched between two sulfur-atom layers by strong covalent bonds. Such strong covalent bonding endows $\mathrm{MoS}_{2}$ with excellent mechanical strength and a high thermal stability up to $1090{ }^{\circ} \mathrm{C}$ in an inert environment. ${ }^{19}$ Additionally, adjacent layers of $\mathrm{MoS}_{2}$ are coupled to each other by weak van der Waals interactions, which enables $\mathrm{MoS}_{2}$ to be created layer by layer and to exhibit diverse novel physical properties.

It is well acknowledged that the photoelectric properties and exciton properties of two-dimensional layered $\mathrm{MoS}_{2}$ depend sensitively on the number of layers, and the interlayer

${ }^{a}$ State Key Laboratory of Superhard Materials, College of Physics, Jilin University, Changchun 130012, China.E-mail: zhouqiang@jlu.edu.cn

${ }^{b}$ State Key Laboratory of Integrated Optoelectronics, College of Electronic Science and Engineering, Jilin University, 2699 Qianjin Street, Changchun 130012, China. E-mail: lugy@jlu.edu.cn

${ }^{c}$ Center for Advanced Optoelectronic Functional Materials Research, Key Laboratory of UV-Emitting Materials and Technology, Northeast Normal University, Ministry of Education, 5268 Renmin Street, Changchun 130024, China interaction plays a crucial role in determining the unique electronic, ${ }^{20}$ optical $^{21}$ and exciton ${ }^{22}$ properties of layered materials in a complex way. Therefore, in this regard, understanding the underlying influence of interlayer interactions on the optical and electrical properties of two-dimensional layered $\mathrm{MoS}_{2}$ is particularly important. However, most of the interlayer interaction-relevant theoretical and experimental studies, such as the investigation regarding the effect of interlayer interactions on the molecular structure, ${ }^{23,24}$ on the band gap, ${ }^{25}$ and on optical properties ${ }^{26}$ are performed on multilayer $\mathrm{MoS}_{2}$ or bulk $\mathrm{MoS}_{2}$, which may introduce some unnecessary interference. Moreover, in spite of some recent investigations on the effects of interlayer interactions on steady-state optical properties of ultrathin $\mathrm{MoS}_{2},{ }^{27}$ the influences of interlayer interactions on transient optical properties, especially on the ultrafast relaxation dynamics of excitons are still unknown, which however are of key importance for realizing potential applications of the novel ultrathin $\mathrm{MoS}_{2}$.

In this contribution, to shed light on the underlying influences of interlayer interactions on the relaxation dynamics of excitons in ultrathin $\mathrm{MoS}_{2}$, we conducted a systematic study by analyzing the discrepancy in relaxation dynamics and photoluminescence only between bilayer $\mathrm{MoS}_{2}$ and monolayer $\mathrm{MoS}_{2}$. The experimental results indicate that the relaxation dynamics and photoluminescence properties of excitons in bilayer $\mathrm{MoS}_{2}$ were significantly different from those in monolayer $\mathrm{MoS}_{2}$ because of the presence of interlayer interactions. For example, in comparison to the monolayer $\mathrm{MoS}_{2}$, a dramatic reduction of photoluminescence quantum efficiency and a remarkably accelerated relaxation rate of A excitons were observed in bilayer $\mathrm{MoS}_{2}$. The possible reasons for this special difference were discussed in terms of the effects of interlayer interactions in detail. 


\section{Experimental}

\subsection{Preparation of two-dimensional layered $\mathrm{MoS}_{2}$ samples}

Monolayer and bilayer $\mathrm{MoS}_{2}$ samples were synthesized on the surface of a quartz substrate by a chemical vapor deposition (CVD) method using a tube furnace with two separate hot zones. ${ }^{28}$ In a typical procedure, two ceramic boats loaded with $700 \mathrm{mg}$ of sulfur powder and $10 \mathrm{mg}$ of $\mathrm{MoO}_{3}$ powder were first placed at the central part of each tube furnace, as shown in Fig. 1(a and b). To provide a favourable growth atmosphere, the quartz tube was vacuumed and then ultra-pure argon gas was flowed into the reaction tube under a low pressure of about 0.4 atm. Then, the $\mathrm{MoO}_{3}$ powder was quickly heated to $850{ }^{\circ} \mathrm{C}$ at a heating rate of $25{ }^{\circ} \mathrm{C} \min ^{-1}$ and the sulfur powder mildly sublimated at a heating rate of $6{ }^{\circ} \mathrm{C} \mathrm{min}^{-1}$ to $200{ }^{\circ} \mathrm{C}$. After the two separate hot zones reached to $850{ }^{\circ} \mathrm{C}$ and $200{ }^{\circ} \mathrm{C}$, it was going to need another $15 \mathrm{~min}$ for $\mathrm{MoS}_{2}$ growth before finally naturally cooling down to room temperature.

\subsection{Characterization}

Raman and photoluminescence spectra were detected using a micro-Raman spectrometer (Horiba-JY T64000) with a $100 \times$ objective in a back-scattering configuration, using a solid state green laser with $532 \mathrm{~nm}$ wavelength as the excitation source. The steady-state absorption spectra were measured using an Ocean Optics QE65000 spectrometer. The layer-dependent signals of relaxation dynamics of excitons were characterized using a self-designed femtosecond transient absorption spectroscopy (fs-TAS) system, as shown in Fig. 2. Our fs-TAS system is composed of a coaxial pump-probe system, white-light probe system and optical imaging system. In brief, a regenerative amplified Ti/sapphire laser (Spectra-Physics) was employed to produce an $800 \mathrm{~nm}$ laser pulse characterized by a 35 fs pulse width and a $1 \mathrm{kHz}$ repetition rate. Then the initial output laser beam was split into two beams: the frequency of one beam was
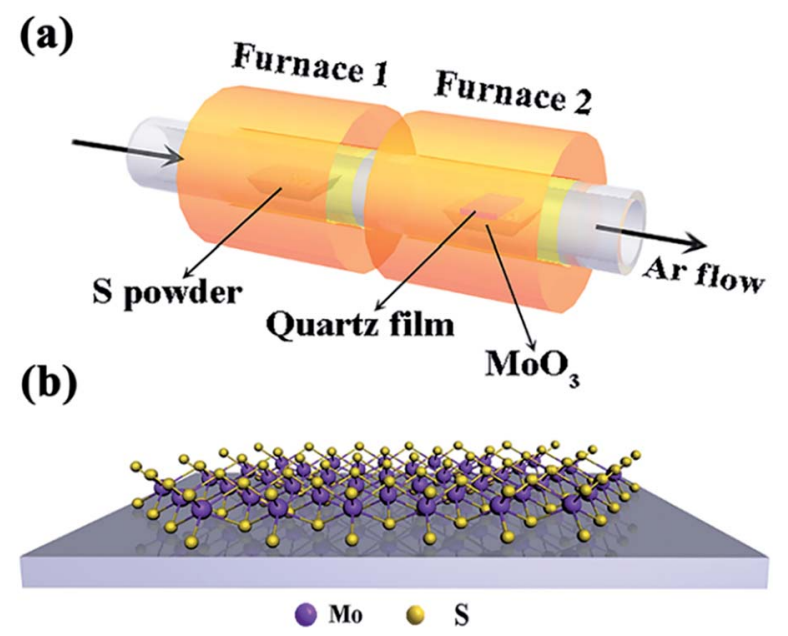

Fig. 1 (a) Schematic illustration of the tube-furnace setup employed in this study for the synthesis of monolayer and bilayer $\mathrm{MoS}_{2}$; (b) a schematic view of the two-dimensional structures of monolayer $\mathrm{MoS}_{2}$ on the substrate. doubled to generate the pump beam (400 nm), and the other beam was focused on a $5 \mathrm{~mm}$ water container to produce white light acting as a probe pulse. Finally, the probe pulse met with the pump beam and remained coaxial with the pump beam. The radii of the focal spots of the pump and probe beams on the sample were measured to be $9 \mu \mathrm{m}$ and $5 \mu \mathrm{m}$ based on the real time observation using an optical microscope. The time delay between the pump and probe beams was achieved with a computer-controlled translation stage (M-405.DG, PI Corp.), and the signal was sent to a lock-in amplifier (SR830, Stanford Co.) to be further processed.

\section{Results and discussion}

Fig. 3(a) shows the optical micrograph of the as-synthesized $\mathrm{MoS}_{2}$ samples and it can be seen that the boundaries of different areas are very clear, and the two large homogeneous regions marked ' 1 ' and ' 2 ' were preliminarily identified to be layered $\mathrm{MoS}_{2}$. And the sizes of the samples at ' 1 ' and ' 2 ' are estimated to be about $130 \mu \mathrm{m}^{2}$ and $450 \mu \mathrm{m}^{2}$ with the assistance of a ruler scale. Moreover, to figure out the accurate layer number of the as-obtained $\mathrm{MoS}_{2}$ samples, Raman spectroscopy was further performed. ${ }^{29}$ Fig. 3(b) shows the Raman spectra of the as-synthesized $\mathrm{MoS}_{2}$ samples located at the regions marked by ' 1 ' and '2' in Fig. 3(a). As can be seen, there are five Raman modes detected in the center of the Brillouin zone at region ' 1 ', namely, shear mode $\left(\mathrm{E}_{2 \mathrm{~g}}^{2}\right)$ at $22.7 \mathrm{~cm}^{-1}$, breathing mode $\left(\mathrm{B}_{2 \mathrm{~g}}^{2}\right)$ at $40.27 \mathrm{~cm}^{-1}$, in-plane vibration mode $\left(\mathrm{E}_{2 \mathrm{~g}}^{1}\right)$ at $383.54 \mathrm{~cm}^{-1}$, outof-plane vibration mode $\left(\mathrm{A}_{1 \mathrm{~g}}\right)$ at $405.46 \mathrm{~cm}^{-1},{ }^{30}$ and the secondorder longitudinal acoustic phonon mode (2LA(M)) at 452.21 $\mathrm{cm}^{-1} \cdot{ }^{31}$ The positions of the peaks corresponding to these five Raman modes are in good agreement with the Raman eigenfrequency of bilayer $\mathrm{MoS}_{2}$ reported in a previous report; ${ }^{32}$ thus region " 1 " was identified to be bilayer $\mathrm{MoS}_{2}$.

Fig. 3(c) shows the schematic diagram of typical bilayer $\mathrm{MoS}_{2}$ and its corresponding characteristic vibration modes. It is clear that the $\mathrm{B}_{2 \mathrm{~g}}^{2}$ mode corresponds to a rigid-layer movement parallel to the $c$ axis, and the $\mathrm{E}_{2 \mathrm{~g}}^{2}$ mode corresponds to a rigid-layer movement perpendicular to the $c$ axis. Both of them exist only in two or more layers, so there should be no $\mathrm{E}_{2 \mathrm{~g}}^{2}$ and $\mathrm{B}_{2 \mathrm{~g}}^{2}$ modes when there is only a single layer of $\mathrm{MoS}_{2}$. Based on the above analysis, it can be seen from Fig. 3(b) that there are only three Raman modes $\left(\mathrm{A}_{1 \mathrm{~g}}, \mathrm{E}_{2 \mathrm{~g}}^{1}\right.$ and $\left.2 \mathrm{LA}(\mathrm{M})\right)$ detected in the Raman spectra for region ' 2 ', and the absence of $E_{2 g}^{2}$ and $B_{2 g}^{2}$ Raman signals implied that the region ' 2 ' is monolayer $\mathrm{MoS}_{2}{ }^{33}$

Fig. 4(a) presents the UV-vis absorption spectra of the monolayer and bilayer $\mathrm{MoS}_{2}$. As can be seen, there are two prominent absorption bands centered at 660 and $618 \mathrm{~nm}$ for both monolayer and bilayer $\mathrm{MoS}_{2}$. By contrast, the intensity of the absorption bands of bilayer $\mathrm{MoS}_{2}$ is much stronger than that of monolayer $\mathrm{MoS}_{2}$. The absorption band centered at 660 $\mathrm{nm}$ is well indexed to A excitons' absorption, while the absorption band around $618 \mathrm{~nm}$ can be indexed to B excitons' absorption. ${ }^{34}$ The absorption of $\mathrm{A}$ and $\mathrm{B}$ excitons is generally believed to be derived from the optical transition from the valence bands $\left(K_{\mathrm{v} 1}\right.$ and $\left.K_{\mathrm{v} 2}\right)$ to the minimum of the conduction band $\left(K_{\mathrm{c}}\right)$ at the $K$ point in the Brillouin zone, respectively, as 


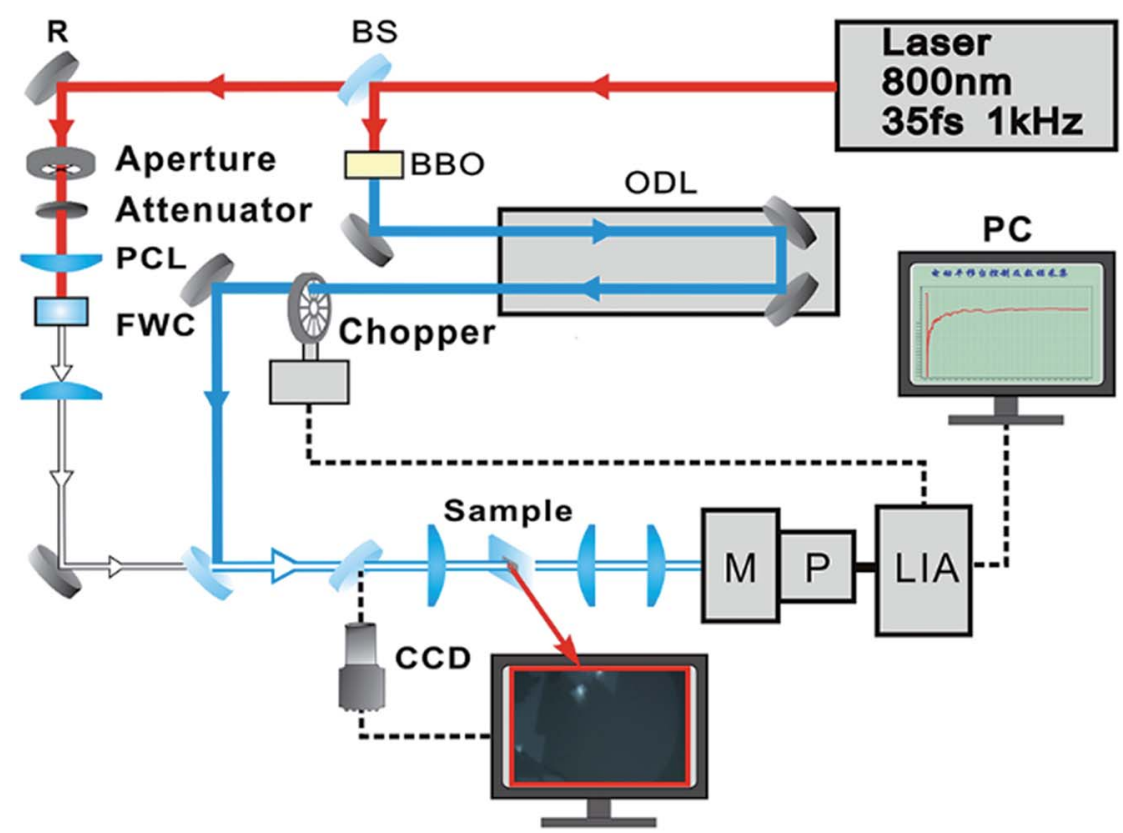

Fig. 2 The schematic diagram of a femtosecond transient absorption spectroscopy system: $\mathrm{BS}_{1}$ and $\mathrm{BS}$, $3: 7$ and $1: 1$ beam splitter; $\mathrm{BBO}, 0.5$ $\mathrm{mm}$-thick $\beta-\mathrm{BaB}_{2} \mathrm{O}_{4}$ crystal; $\mathrm{ODL}$, optical delay line; $\mathrm{R}$, reflector; $\mathrm{CL}$, convex lens; $\mathrm{FWC}$, flow water cell; $\mathrm{M}$, three grating monochromator; $\mathrm{P}$, photomultiplier; LIA, lock-in amplifier.

schematically shown in Fig. 4(b). In addition, the energy difference between $K_{\mathrm{v} 1}$ and $K_{\mathrm{v} 2}$ bands stems from the splitting of the maximum valence band, ${ }^{35,36}$ which is believed to be caused by the combined effects of interlayer interactions and spin-orbit coupling for bilayer $\mathrm{MoS}_{2}$ and only the effect of spinorbit coupling for monolayer $\mathrm{MoS}_{2}{ }^{22,37}$

In spite of the above well-discussed different stable-state absorption properties of excitons caused by interlayer interactions, the influence of interlayer interactions on the relaxation dynamics of excitons in $\mathrm{MoS}_{2}$ still remains unknown and needs to be further addressed. Thus the influence of the interlayer interaction on exciton relaxation dynamics was further investigated with the assistance of our self-designed femtosecond transient absorption spectroscopy (fs-TAS) platform. Since the resonance absorption of A excitons for both monolayer and bilayer $\mathrm{MoS}_{2}$ was around $660 \mathrm{~nm}$ as shown in

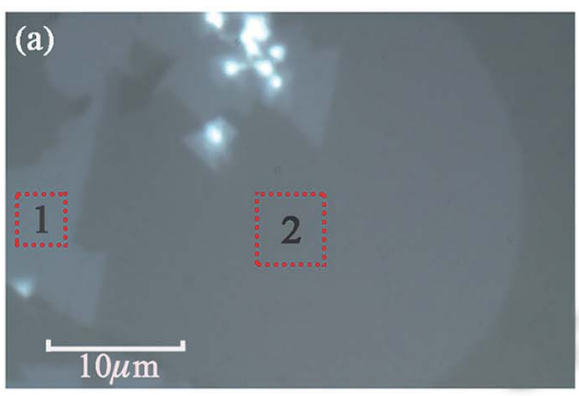

(c)

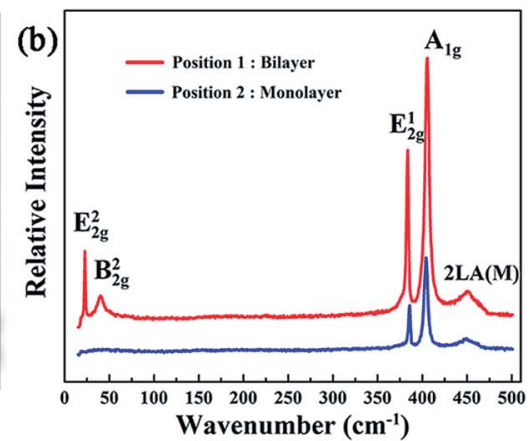

Wavenumber $\left(\mathrm{cm}^{-1}\right)$

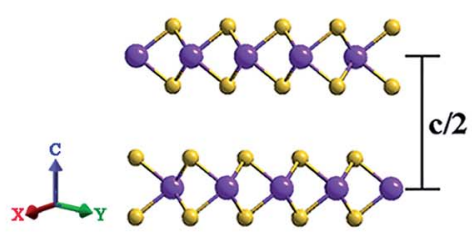

Mo

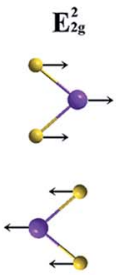

OS

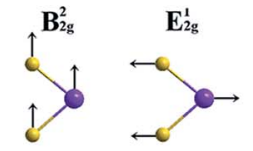

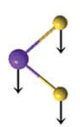<smiles>COB(O)[Hg]</smiles><smiles>COCOCCOCCOCCO</smiles>

Fig. 3 (a) Optical micrograph of layered $\mathrm{MoS}_{2}$ samples deposited on the quartz substrate; (b) Raman spectra of the as-synthesized MoS 2 samples in the regions marked ' 1 ' and ' 2 ' in (a); (c) schematic diagrams of the lattice structure (left side) in out-of-plane directions of MoS $_{2}$ and its corresponding characteristic Raman vibration modes (right side). 
(a)

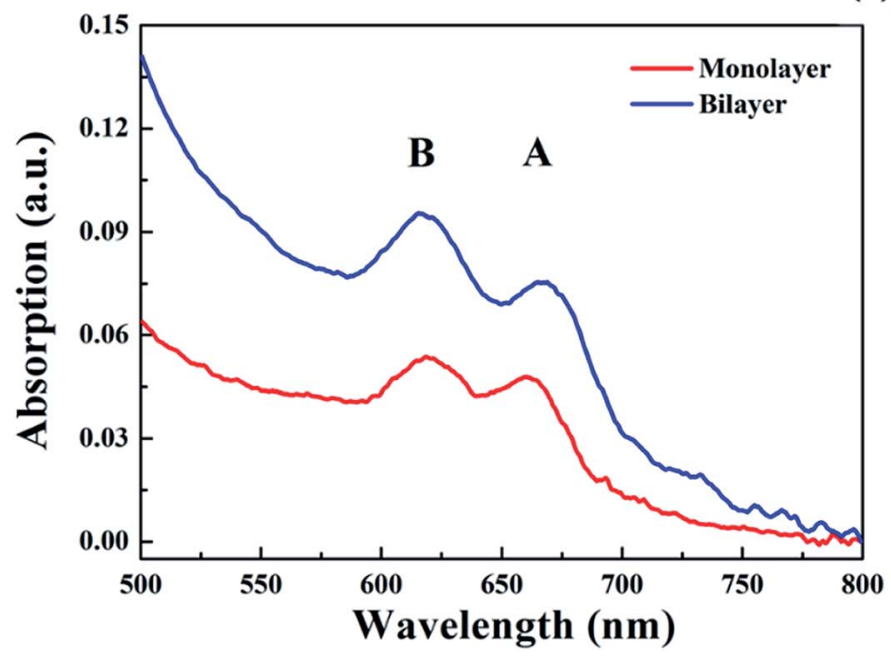

(b)

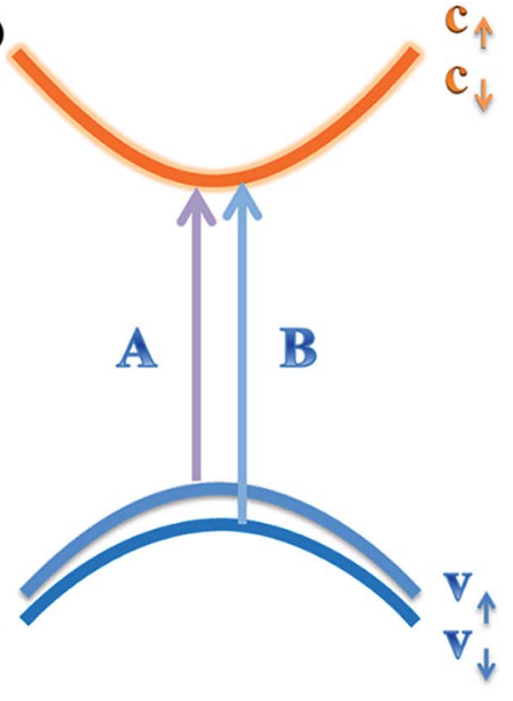

Fig. 4 (a) The UV-vis absorption spectra of the as-grown monolayer and bilayer $\mathrm{MoS}_{2}$ after being returned to zero at $800 \mathrm{~nm}$; (b) simplified band structure of $\mathrm{MOS}_{2}$ at the $K$ point in the Brillouin zone.

the UV-vis absorption spectra in Fig. 4(a), the probe laser wavelength was chosen to be $660 \mathrm{~nm}$ during the fs-TAS measurements. Besides, to guarantee the formation of photoexcited excitons at the A exciton level, the pump laser wavelength was set at $400 \mathrm{~nm}$. And the pump intensity was selected to be $2 \mu \mathrm{J} \mathrm{cm}^{-2}$ according to the linear relationship between the signal intensity and pump intensity (Fig. 5) to get strong and steady signals.

Fig. 6(a) displays the relaxation dynamics curves of A excitons for monolayer and bilayer $\mathrm{MoS}_{2}$. It is noteworthy that the relaxation time of bilayer $\mathrm{MoS}_{2}$ was much shorter than that of monolayer $\mathrm{MoS}_{2}$, which implies that the whole exciton relaxation became faster in the presence of interlayer interactions. Furthermore, the decay curves of monolayer and bilayer $\mathrm{MoS}_{2}$ were fitted separately and the fitting parameters for the decay curves are summarized in Table 1 . As can be seen, both the relaxation dynamics signals of monolayer and bilayer $\mathrm{MoS}_{2}$ exhibit multi-exponential decays and can be fitted very well using a three exponential Gaussian response function of $a_{1^{-}}$ $\exp \left(-t / \tau_{1}\right)+a_{2} \exp \left(-t / \tau_{2}\right)+a_{3} \exp \left(-t / \tau_{3}\right)$, where $\tau_{1}<\tau_{2}<\tau_{3}$, which implies that multiple relaxation processes were involved. For monolayer $\mathrm{MoS}_{2}$, the decay was characterized by a fast decay time constant $\left(\tau_{1}\right)$ of $3.3 \pm 0.1 \mathrm{ps}$, an intermediate decay time constant $\left(\tau_{2}\right)$ of $82.9 \pm 5.6 \mathrm{ps}$ and a slow decay time constant $\left(\tau_{3}\right)$ of $1053.9 \pm 26.6 \mathrm{ps}$, the proportion of the above three time constants was $35 \%, 20 \%$ and $45 \%$ in turn. Moreover, the relaxation lifetime of monolayer $\mathrm{MoS}_{2}$ is $489.5 \pm 13.1 \mathrm{ps}$, which is close to the values in the literature, ${ }^{38}$ while for bilayer $\mathrm{MoS}_{2}$, the decay time constant was composed of a fast decay $\left(\tau_{1}\right)$ of 3.7 $\pm 0.1 \mathrm{ps}$ (31\% weight), an intermediate decay $\left(\tau_{2}\right)$ of $42.4 \pm 0.8$ ps $(51 \%$ weight $)$, and a slow decay $\left(\tau_{3}\right)$ of $714.4 \pm 25.1$ ps $(18 \%$ weight), and the relaxation lifetime is $150.7 \pm 4.9 \mathrm{ps}$. The obviously changed proportion of the decay time constant and the significantly reduced relaxation lifetime for bilayer $\mathrm{MoS}_{2}$ imply that interlayer interactions have a significant effect on the relaxation characteristics of excitons.
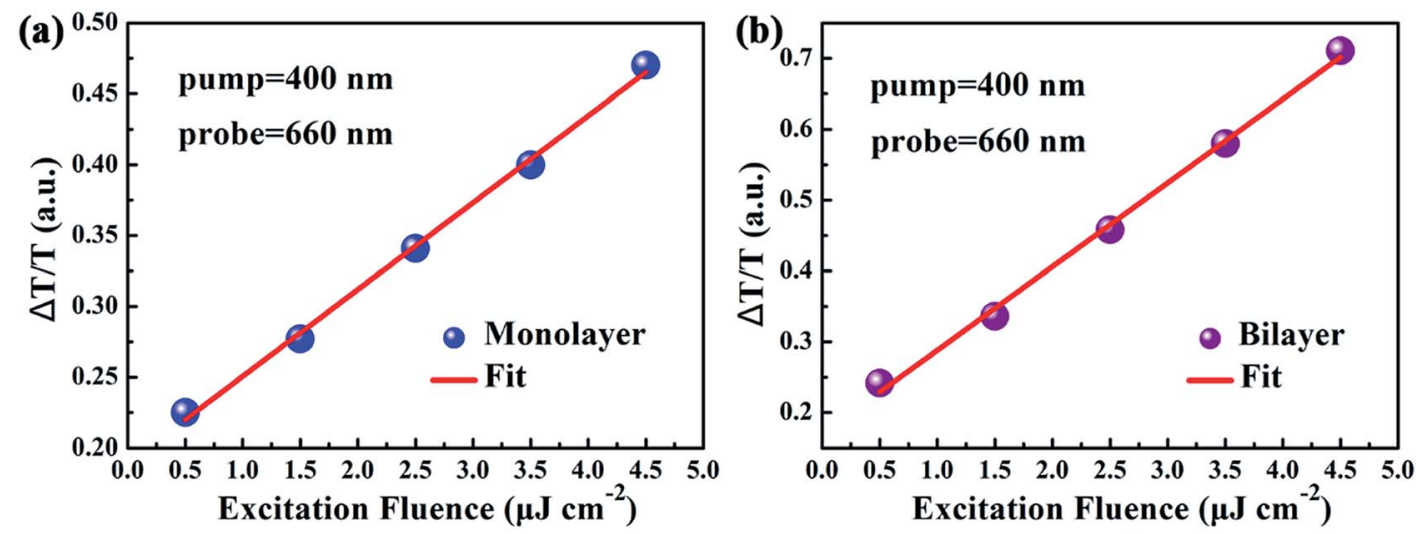

Fig. 5 Signal intensity of $\Delta T / T$ at zero time delay as a function of excitation intensity for (a) monolayer and (b) bilayer MoS 2 with a pump wavelength of $400 \mathrm{~nm}$ and a probe wavelength of $660 \mathrm{~nm}$. The circles denote the experimental data, while the solid lines denote the linear fitting data. 


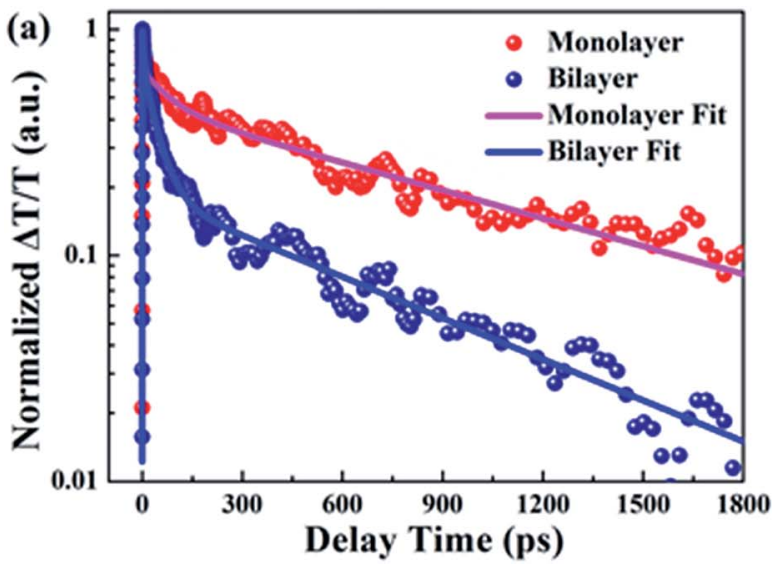

(b)

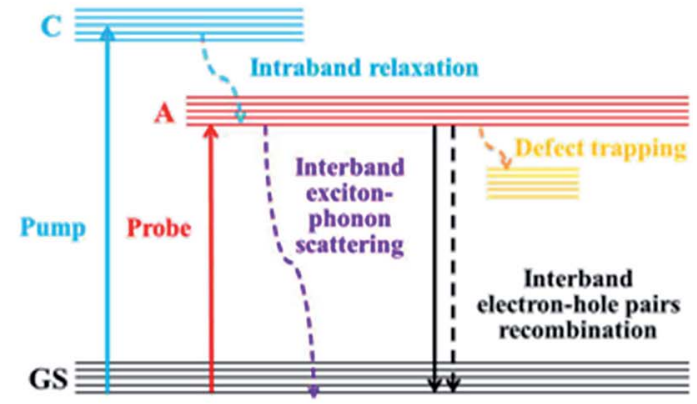

Monolayer and Bilayer $\mathrm{MoS}_{2}$

Fig. 6 (a) Experimental (solid circles) and fitted (thick lines) data for normalized transient absorption relaxation dynamics curves of monolayer and bilayer $\mathrm{MoS}_{2}$. All the relaxation dynamics curves were normalized at time zero, being fitted with a three exponential function $/(t)=a_{1}$ exp $(-t /$ $\left.\tau_{1}\right)+a_{2} \exp \left(-t / \tau_{2}\right)+a_{3} \exp \left(-t / \tau_{3}\right)$; (b) schematic summary of relaxation processes in monolayer and bilayer $\mathrm{MoS}_{2}$.

Table 1 Fitting parameters for decay curves in Fig. 6(a) using a Gaussian response function with the three-exponential decay functions of $I(t)=$ $a_{1} \exp \left(-t / \tau_{1}\right)+a_{2} \exp \left(-t / \tau_{2}\right)+a_{3} \exp \left(-t / \tau_{3}\right)$ with the fitting uncertainty. The percentage in the brackets represents the proportion of $\tau_{1}$, $\tau_{2}$ and $\tau_{3}$

\begin{tabular}{lllll}
\hline & $\tau_{1}(\mathrm{ps})$ & $\tau_{2}(\mathrm{ps})$ & $\tau_{3}(\mathrm{ps})$ & Lifetime $(\mathrm{ps})$ \\
\hline Monolayer & $3.3 \pm 0.1(35 \%)$ & $82.9 \pm 5.6(20 \%)$ & $1053.9 \pm 26.6(45 \%)$ & $489.5 \pm 13.1$ \\
Bilayer & $3.7 \pm 0.1(31 \%)$ & $42.4 \pm 0.8(51 \%)$ & $717.4 \pm 25.1(18 \%)$ & $150.7 \pm 4.9$
\end{tabular}

To shed light on the underlying mechanism for the above dramatic reduction of the exciton relaxation time observed in bilayer $\mathrm{MoS}_{2}$, the transient absorption decay dynamics of monolayer and bilayer $\mathrm{MoS}_{2}$ were further characterized at different pump intensities, and the normalized dynamics curves are shown in Fig. 7. It can be seen that no significant dynamics dependences on the pump intensity were observed in either monolayer or bilayer $\mathrm{MoS}_{2}$, which indicates that the fast decay component was not the exciton-exciton annihilation process in this work. Since shallow and deep traps usually exist in $\mathrm{MoS}_{2}$ nanoclusters, ${ }^{39}$ and the fast decay time $\left(\tau_{1}\right)$ obtained here is similar to the time scale of defect trapping; ${ }^{40}$ thus the fast decay component observed here was closely related to fast defect trapping. In addition, considering the characteristics of ultrathin $\mathrm{MoS}_{2}$, the exciton-phonon interaction is going to be very significant in monolayer and bilayer $\mathrm{MoS}_{2}$. Since the intermediate decay time constant $\left(\tau_{2}\right)$ in our work was of the same order as the exciton-phonon scattering time reported in a recent study, ${ }^{40}$ the intermediate decay observed here might be attributed to the exciton-phonon scattering. The slowest decay process that lasted for hundreds of picoseconds $\left(\tau_{3}\right)$ is generally believed to be derived from the recombination of electrons and holes. The schematic of the relaxation processes involved in monolayer and bilayer $\mathrm{MoS}_{2}$ is shown in Fig. 6(b).
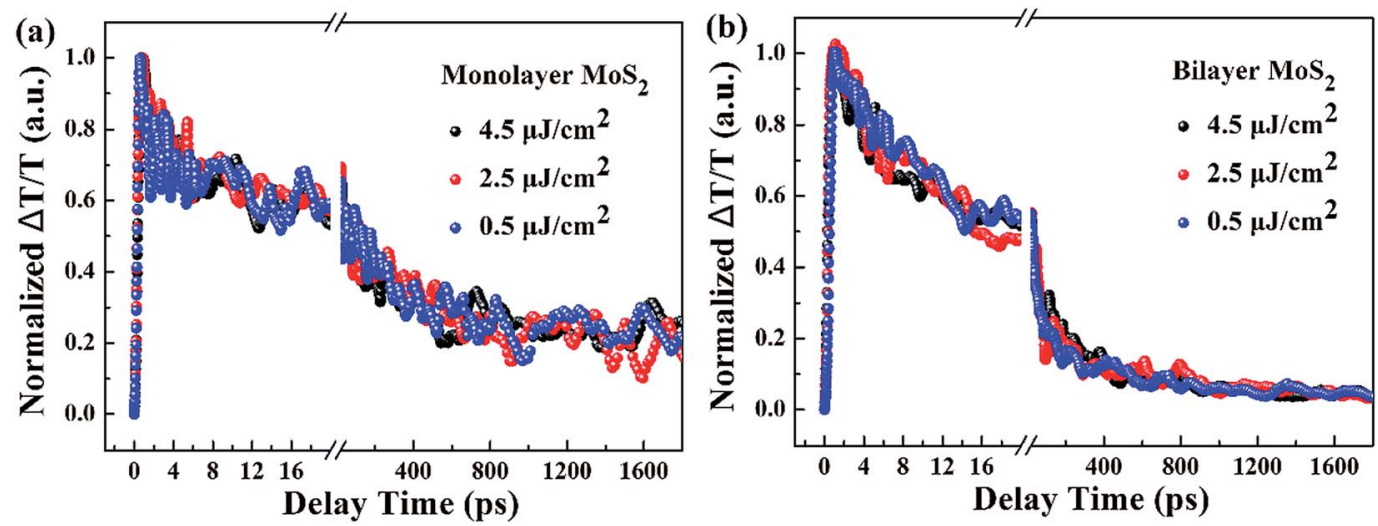

Fig. 7 Normalized transient absorption decay dynamics curves of (a) monolayer and (b) bilayer $\mathrm{MoS}_{2}$ with a pump wavelength of $400 \mathrm{~nm}$ and a probe wavelength of $660 \mathrm{~nm}$ at different pump intensities. 
It is worth noting that unlike the similar values of $\tau_{1}$, the values of $\tau_{2}$ and $\tau_{3}$ for bilayer $\mathrm{MoS}_{2}$ were both significantly lower than those for monolayer $\mathrm{MoS}_{2}$. This dramatic enhancement of the exciton relaxation rate observed in bilayer $\mathrm{MoS}_{2}$ might be understood by the following aspects. Firstly, as can be seen from Fig. 3(b), the additional Raman vibration modes $\left(\mathrm{B}_{2 \mathrm{~g}}^{2}\right.$ and $\mathrm{E}_{2 \mathrm{~g}}^{2}$ ) and the stronger Raman vibration mode of $\mathrm{A}_{1 \mathrm{~g}}$ in bilayer $\mathrm{MoS}_{2}$ imply that the vibration of the atoms and lattice was enhanced due to the presence of interlayer interactions in comparison to monolayer $\operatorname{MoS}_{2} \cdot{ }^{41}$ And the enhanced vibration of the lattice caused by the interlayer interaction will facilitate the exciton-phonon scattering in bilayer $\mathrm{MoS}_{2}$, resulting in a shorter intermediate decay time $\tau_{2}$.

Secondly, since monolayer $\mathrm{MoS}_{2}$ is a direct band-gap semiconductor, most of the electrons and holes are located on the conduction band minimum (CBM) and valence band maximum (VBM) at the $K$ point, respectively; thus the radiative recombination of electrons and holes happens very easily, which can be verified by the extremely strong photoluminescence (PL) intensity of monolayer $\mathrm{MoS}_{2}$ as shown in Fig. 8. Therefore, the main recombination process of electrons and holes in monolayer $\mathrm{MoS}_{2}$ is radiative recombination. However, bilayer $\mathrm{MoS}_{2}$ is an indirect band-gap semiconductor, the photon energy (3.1 eV) employed here will pump the initial carriers to a higher energy level, and the fast cooling of the carriers would let the electrons and holes locate on the CBM at the $K$ point and the VBM at the $\Gamma$ point, ${ }^{2}$ respectively. Thus, the radiative recombination of electrons and holes in bilayer $\mathrm{MoS}_{2}$ requires the assistance of phonons, ${ }^{42}$ which will significantly suppress the radiative relaxation process in bilayer $\mathrm{MoS}_{2}$. Moreover, many previous reports have proved that the interlayer interaction can redistribute the charges, which gives rise to a local electric field at the interfacial region in bilayer $\mathrm{MoS}_{2}$. After being excited by the pump beam, the electrons will accumulate at the interfacial surfaces, while the holes will thus be pushed away from the interface due to the local electric field, which will lead to spatial separation of electrons and holes. And the reduction of the

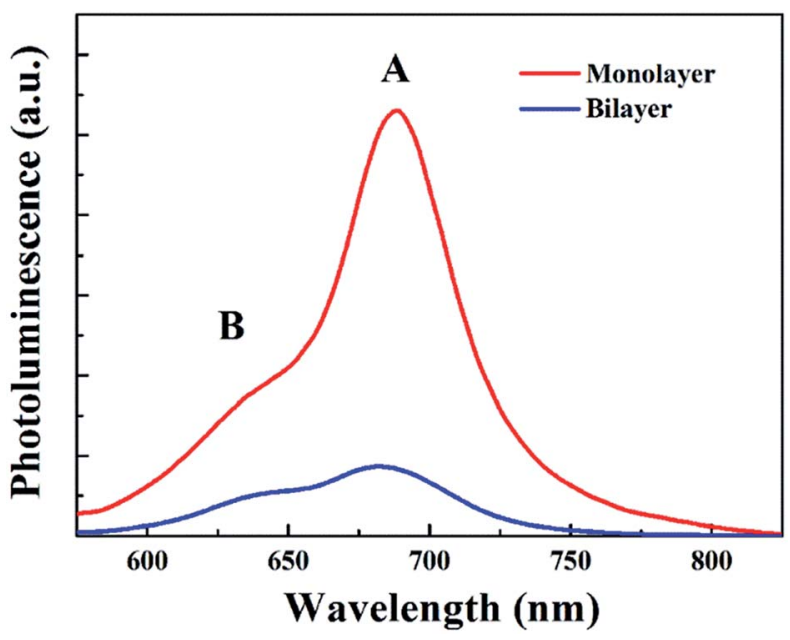

Fig. 8 Photoluminescence $(\mathrm{PL})$ spectra of monolayer and bilayer $\mathrm{MoS}_{2}$. overlap between electron and hole wavefunctions will make the relaxation of electrons and holes through radiative recombination difficult, resulting in the decrease of fluorescence quantum yield. ${ }^{27}$ This can also be seen in the PL spectra shown in Fig. 8. It can be seen from Fig. 8 that the PL intensity of A excitons reduced dramatically in bilayer $\mathrm{MoS}_{2}$, which suggests that the radiative recombination becomes negligible in bilayer $\mathrm{MoS}_{2}$ compared to monolayer $\mathrm{MoS}_{2}$. Therefore, the prominent recombination process of electrons and holes in bilayer $\mathrm{MoS}_{2}$ is non-radiative recombination. Furthermore, it is well known that the decay time of non-radiative recombination is usually shorter than that of radiative recombination. ${ }^{\mathbf{4 3 , 4 4}}$ Therefore the decay time $\tau_{3}$ of bilayer $\mathrm{MoS}_{2}$ is much shorter than that of monolayer $\mathrm{MoS}_{2}$. Given the above, it therefore can be concluded that the dramatic enhancement of the whole relaxation rate of $\mathrm{A}$ excitons for bilayer $\mathrm{MoS}_{2}$ can be explained by the combined effects of the enhanced exciton-phonon scattering and reduced radiative relaxation induced by interlayer interactions.

\section{Conclusions}

In summary, we performed a detailed investigation into the underlying influence of interlayer interactions on the relaxation dynamics of excitons in ultrathin $\mathrm{MoS}_{2}$ by analyzing the discrepancy in relaxation dynamics and photoluminescence properties only between bilayer $\mathrm{MoS}_{2}$ and monolayer $\mathrm{MoS}_{2}$. On the basis of thoughtful analysis about the obtained experimental data, both monolayer and bilayer $\mathrm{MoS}_{2}$ samples exhibit multiple relaxation processes involving the defect trapping, the exciton-phonon scattering, and the recombination process of electrons and holes. Moreover, the characterization results manifest that the relaxation rate of A excitons was significantly accelerated in bilayer $\mathrm{MoS}_{2}$ compared to monolayer $\mathrm{MoS}_{2}$, which is due to the combined effects of the enhanced excitonphonon scattering and reduced radiative relaxation induced by interlayer interactions.

\section{Conflicts of interest}

There are no conflicts to declare.

\section{Acknowledgements}

We are grateful to Prof. Yisong Zheng and Prof. Jianzhong Zhang for many fruitful discussions. We also thank Prof. Kai Wang and researcher Guohui Lu for their help during the experimental research. This work was supported by the financial support from the National Natural Science Foundation of China (no. 11274137, 11574112, 11474127, 51304080).

\section{References}

1 K. F. Mak, C. Lee, J. Hone, J. Shan and T. F. Heinz, Phys. Rev. Lett., 2010, 105, 136805.

2 X. M. Dou, K. Ding, D. S. Jiang and B. Q. Sun, ACS Nano, 2014, 8, 7458-7464. 
3 A. R. Klots, A. K. M. Newaz, B. Wang, D. Prasai, H. Krzyzanowska, J. H. Lin, D. Caudel, N. J. Ghimire, J. Yan, B. L. Ivanov, K. A. Velizhanin, A. Burger, D. G. Mandrus, N. H. Tolk, S. T. Pantelides and K. I. Bolotin, Sci. Rep., 2014, 4, 6608.

4 N. Saigal, V. Sugunakar and S. Ghosh, Appl. Phys. Lett., 2016, 108, 132105.

5 T. Cao, G. Wang, W. P. Han, H. Q. Ye, C. R. Zhu, J. R. Shi, Q. Niu, P. H. Tan, E. Wang, B. L. Liu and J. Feng, Nat. Commun., 2012, 3, 887.

6 K. F. Mak, K. L. He, J. Shan and T. F. Heinz, Nat. Nanotechnol., 2012, 7, 494-498.

7 G. L. Frey, K. J. Reynolds, R. H. Friend, H. Cohen and Y. Feldman, J. Am. Chem. Soc., 2003, 125, 5998-6007.

8 G. J. Choi, Q. V. Le, K. S. Choi, K. C. Kwon, H. W. Jang, J. S. Gwag and S. Y. Kim, Adv. Mater., 2017, 29, 1702598.

9 K. P. Guo, C. F. Si, C. Han, S. H. Pan, G. Chen, Y. Q. Zheng, W. Q. Zhu, J. H. Zhang, C. Sun and B. Wei, Nanoscale, 2017, 9, 14602-14611.

10 Y. X. Deng, Z. Luo, N. J. Conrad, H. Liu, Y. J. Gong, S. Najmaei, P. M. Ajayan, J. Lou, X. F. Xu and P. D. Ye, ACS Nano, 2014, 8, 8292-8299.

11 Y. Niu, R. Frisenda, S. A. Svatek, G. Orfila, F. Gallego, P. Gant, N. Agrait, C. Leon, A. Rivera-Calzada, D. P. De Lara, J. Santamaria and A. Castellanos-Gomez, 2D Mater., 2017, 4, 034002.

12 Z. Li, J. H. Chen, R. Dhall and S. B. Cronin, 2D Mater., 2017, 4, 015004.

13 X. Gu, W. Cui, H. Li, Z. W. Wu, Z. Y. Zeng, S. T. Lee, H. Zhang and B. Q. Sun, Adv. Energy Mater., 2013, 3, 1262-1268.

14 M. L. Tsai, S. H. Su, J. K. Chang, D. S. Tsai, C. H. Chen, C. I. Wu, L. J. Li, L. J. Chen and J. H. He, ACS Nano, 2014, 8, 8317-8322.

15 A. Capasso, F. Matteocci, L. Najafi, M. Prato, J. Buha, L. Cina, V. Pellegrini, A. Di Carlo and F. Bonaccorso, Adv. Energy Mater., 2016, 6, 1600920.

16 G. H. Lee, Y. J. Yu, X. Cui, N. Petrone, C. H. Lee, M. S. Choi, D. Y. Lee, C. Lee, W. J. Yoo, K. Watanabe, T. Taniguchi, C. Nuckolls, P. Kim and J. Hone, ACS Nano, 2013, 7, 79317936.

17 B. Radisavljevic, A. Radenovic, J. Brivio, V. Giacometti and A. Kis, Nat. Nanotechnol., 2011, 6, 147-150.

18 H. Ji, H. Yi, J. Seok, H. Kim, Y. H. Lee and S. C. Lim, Nanoscale, 2018, 10, 10856-10862.

19 S. Bertolazzi, J. Brivio and A. Kis, ACS Nano, 2011, 5, 97039709.

20 W. S. Yun, S. W. Han, S. C. Hong, I. G. Kim and J. D. Lee, Phys. Rev. B, 2012, 85, 033305.

21 A. Splendiani, L. Sun, Y. B. Zhang, T. S. Li, J. Kim, C. Y. Chim, G. Galli and F. Wang, Nano Lett., 2010, 10, 1271-1275.
22 T. Cheiwchanchamnangij and W. R. L. Lambrecht, Phys. Rev. $B, 2012,85,205302$.

23 Y. L. Yan, F. F. Li, Y. B. Gong, M. G. Yao, X. L. Huang, X. P. Fu, B. Han, Q. Zhou and T. Cui, J. Phys. Chem. C, 2016, 120, 24992-24998.

24 X. M. Dou, K. Ding, D. S. Jiang, X. F. Fan and B. Q. Sun, ACS Nano, 2016, 10, 1619-1624.

25 S. W. Han, H. Kwon, S. K. Kim, S. Ryu, W. S. Yun, D. H. Kim, J. H. Hwang, J. S. Kang, J. Baik, H. J. Shin and S. C. Hong, Phys. Rev. B, 2011, 84, 045409.

26 A. F. Rigos, H. M. Hill, Y. L. Li, A. Chernikov and T. F. Heinz, Nano Lett., 2015, 15, 5033-5038.

27 J. G. Kim, W. S. Yun, S. Jo, J. Lee and C. H. Cho, Sci. Rep., 2016, 6, 29813.

28 Y. H. Lee, X. Q. Zhang, W. J. Zhang, M. T. Chang, C. T. Lin, K. D. Chang, Y. C. Yu, J. T. W. Wang, C. S. Chang, L. J. Li and T. W. Lin, Adv. Mater., 2012, 24, 2320-2325.

29 T. Sekine, K. Uchinokura, T. Nakashizu, E. Matsuura and R. Yoshizaki, J. Phys. Soc. Jpn., 1984, 53, 811-818.

30 T. Wieting and J. Verble, Phys. Rev. B, 1971, 3, 4286-4292.

31 H. Li, Q. Zhang, C. C. R. Yap, B. K. Tay, T. H. T. Edwin, A. Olivier and D. Baillargeat, Adv. Funct. Mater., 2012, 22, 1385-1390.

32 Y. Zhao, X. Luo, H. Li, J. Zhang, P. T. Araujo, C. K. Gan, J. Wu, H. Zhang, S. Y. Quek and M. S. Dresselhaus, Nano Lett., 2013, 13, 1007-1015.

33 H. Zeng, B. Zhu, K. Liu, J. Fan, X. Cui and Q. Zhang, Phys. Rev. B, 2012, 86, 241301.

34 R. Coehoorn, C. Haas and R. De Groot, Phys. Rev. B, 1987, 35, 6203-6206.

35 B. Evans and P. Young, Proc. Phys. Soc., 1967, 91, 475-482.

36 J. Wilson and A. Yoffe, Adv. Phys., 1969, 18, 193-335.

37 R. F. Frindt, Phys. Rev., 1965, 140, A536-A539.

38 L. Yang, D. Zhong, J. Y. Zhang, Z. P. Yan, S. F. Ge, P. W. Du, J. Jiang, D. Sun, X. J. Wu, Z. Y. Fan, S. A. Dayeh and B. Xiang, ACS Nano, 2014, 8, 6979-6985.

39 R. Doolen, R. Laitinen, F. Parsapour and D. F. Kelley, J. Phys. Chem. B, 1998, 102, 3906-3911.

40 T. Korn, S. Heydrich, M. Hirmer, J. Schmutzler and C. Schuller, Appl. Phys. Lett., 2011, 99, 102109.

41 C. Lee, H. Yan, L. E. Brus, T. F. Heinz, J. Hone and S. Ryu, ACS Nano, 2010, 4, 2695-2700.

42 H. Dery and Y. Song, Phys. Rev. B, 2015, 92, 125431.

43 L. Y. Pan, Y. L. Zhang, H. Y. Wang, H. Liu, J. S. Luo, H. Xia, L. Zhao, Q. D. Chen, S. P. Xu, B. R. Gao, L. M. Fu and H. B. Sun, Nanoscale, 2011, 3, 2882-2888.

44 M. Palummo, M. Bernardi and J. C. Grossman, Nano Lett., 2015, 15, 2794-2800. 